\title{
Correction: The lipogenic LXR-SREBF1 signaling pathway controls cancer cell DNA repair and apoptosis and is a vulnerable point of malignant tumors for cancer therapy
}

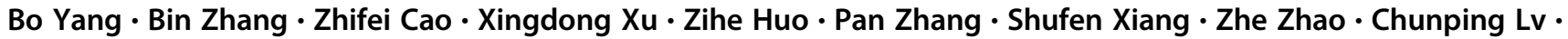 \\ Mei Meng · Gaochuan Zhang · Liang Dong · Shucheng Shi · Lan Yang • Quansheng Zhou
}

Published online: 6 April 2020

(c) The Author(s), under exclusive licence to ADMC Associazione Differenziamento e Morte Cellulare 2020

Correction to: Cell Death \& Differentiation https://doi.org/10.1038/s41418-020-0514-3

The affiliation details appeared incorrectly in this article. The correct affiliation details for these authors are given below:

Bo Yang ${ }^{1 \S}$, Bin Zhang ${ }^{2,3 \S}$, Zhifei $\mathrm{Cao}^{2,4 \S}$, Xingdong $\mathrm{Xu}^{5}$, Zihe $\mathrm{Huo}^{2}$, Pan Zhang ${ }^{2}$, Shufen Xiang ${ }^{2}$, Zhe Zhao ${ }^{2}$, Chunping $\mathrm{Lv}^{2}$, Mei Meng ${ }^{2}$, Gaochuan Zhang ${ }^{6}$, Liang Dong ${ }^{7}$, Shucheng Shi ${ }^{1}$, Lan Yang ${ }^{1}$, Quansheng Zhou ${ }^{2 *}$

1. Department of General Surgery, The Third Affiliated Hospital of Soochow University; The First People's Hospital of Changzhou, Changzhou 213003, PR China

2. Cyrus Tang Hematology Center, Jiangsu Institute of Hematology, Soochow University; State Key Laboratory of Radiation Medicine; Key Laboratory of Thrombosis and Hemostasis, Ministry of Health; 2011 Collaborative Innovation Center of Hematology, Soochow University, Suzhou 215123, Jiangsu, PR China
3. Center of Systems Medicine, Institute of Basic Medical Sciences, Chinese Academy of Medical Sciences \& Peking Union Medical College, 100005 Beijing, China; Suzhou Institute of Systems Medicine, Suzhou 215123, PR China

4. Department of Pathology, The Second Affiliated Hospital of Soochow University, Suzhou 215004, Jiangsu, PR China

5. Department of General Surgery, The People's Hospital of China, Three Gorges University; The First People's Hospital of Yichang, Yichang 443000, PR China

6. Department of Bioinformatics, College of Basic Medical Science, Soochow University, Suzhou 215123, Jiangsu, PR China

7. Department of Pathology, College of Basic Medical Science, Soochow University, Suzhou 215123, Jiangsu, PR China

The online html and PDF have been updated accordingly. 